\title{
Negative Regulation of G1 to S Transition
}

National Cancer Institute

\section{Source}

National Cancer Institute. Negative Regulation of G1 to S Transition. NCI Thesaurus.

Code C21230.

G1-S Arrest consists of cellular biochemical mechanisms, responsive to diverse governing conditions (DNA damage, contact inhibition, growth factors, etc.), that control cellular commitment at the G1 restriction point to DNA replication and the proliferative cycle. G1 to $S$ is controlled by de/phosphorylated cyclin/CDK complexes. During G1, cycD/CDK phosphorylation of Rb/HDAC repressor permits E2F/DP1 transcription of genes that promote the G1/S switch and DNA replication. p53 induces p21 inhibition of cycD/CDK; Mdm2 inhibits p53/p21. TGF-b induces p15 inhibition of cycD/CDK. cAMP induces p27 inhibition of cycD/CDK. GSK3b induction of cycD degradation, and INK4, Kip, and Cdc25A regulate cycD/CDK activity. 